\title{
From laboratory to environmental conditions: a new approach for chemical's biodegradability assessment
}

\author{
Brillet François $^{1} \cdot$ Maul Armand $^{2} \cdot$ Durand Marie-José $^{1} \cdot$ Gérald Thouand $^{1}$
}

\begin{abstract}
With thousands of organic chemicals released every day into our environment, Europe and other continents are confronted with increased risk of health and environmental problems. Even if a strict regulation such as REgistration, Authorization and restriction of CHemicals (REACH) is imposed and followed by industry to ensure that they prove the harmlessness of their substances, not all testing procedures are designed to cope with the complexity of the environment. This is especially true for the evaluation of persistence through biodegradability assessment guidelines. Our new approach has been to adapt "in the lab" biodegradability assessment to the environmental conditions and model the probability for a biodegradation test to be positive in the form of a logistic function of both the temperature and the viable cell density. Here, a proof of this new concept is proposed with the estab-lishment of tri-dimensional biodegradability profiles of six chemicals (sodium benzoate, 4-nitrophenol, diethylene gly-col, 2,4,5-trichlorophenol, atrazine, and glyphosate) between 4 to $30{ }^{\circ} \mathrm{C}$ and 10 to $10^{8}$ cells $\mathrm{ml}$ ${ }^{-1}$ as can be found in environmental compartments in time and space. The results show a significant increase of the predictive power of existing screening lab-scale tests designed for soluble substances. This strategy can be complementary to those current testing
\end{abstract}

Gérald Thouand

gerald.thouand@univ-nantes.fr

1 Université de Nantes, UMR CNRS 6144 GEPEA, IUT Génie Biologique, La Roche sur Yon, France

2 Université de Lorraine, LIEC-UMR CNRS, 7360 Metz, France strategies with the creation of new indicators to quantify environmental persistence using lab-scale tests.

Keywords Biodegradability assessment $\cdot$ Probability of biodegradation $\cdot$ OECD screening tests

\section{Introduction}

According to the American database CHEMCATS, millions of chemicals are commercially available and ubiquitous in our lives. Minimizing the impact of potential pollution is a necessity for environmental protection agencies, researchers, industry, and society as a whole. In Europe, for example, REgistration, Authorization and restriction of CHemicals (REACH) regulations require a biodegradability evaluation of high-production-volume organic chemicals (UE, 2006). The stakes are high; in addition to the substance-specific properties, the fate of each chemical in the environment differs according to its terms of use and mode of discharge (Bartholomew and Pfaender 1983; Federle et al. 1997; Vink and Van Der Zee 1997). In silico models have been developed (Raymond et al. 2001) and are available under REACH regulations (Pizzo et al. 2013), but the prediction of overall persistence with multimedia fate models remains limited to existing data and may be not adapted to assess environmental persistence as a function of both chemical intrinsic properties and environmental conditions (Howard et al. 1992; Fenner et al. 2004; Aronson et al. 2006). Indeed, the various environmental microbial communities, which are potentially involved in the biodegradation of chemicals, are different from each other (Forney et al. 2001; Martiny et al. 2006) and are also subject to the variation of environmental parameters associated with the conditions of in situ life (Zogg et al. 1997; Ranjard et al. 2013). Faced with the impossibility to assess biodegradability 
in real conditions (Mackay and Webster 2005), microbial communities are taken from a selected environment and used in vitro under laboratory conditions (Nyholm 1991). In REACH, Ready Biodegradation Tests (RBT) (OECD 1992) are at the basis of the integrated testing strategy on biodegradation (ECHA 2011; ECHA R.7B 2014). Dedicated to the screening stage, they are individually designed to cope with the physicochemical properties of tested substances (e.g., poorly soluble or volatile). They are cheap and easy to use but give point data limited to one specific type of environment, which leads to a variable and restricted view of biodegradation (Thouand et al. 1995; van Ginkel et al. 1995; Vazquez-Rodriguez et al. 2012; Goodhead et al. 2014). Despite this uncertainty, a positive RBT result can be used to classify the tested substance as readily biodegradable (Ahtiainen et al. 2003; ECHA 2014). Simulation tests (e.g., OECD 314 (OECD 2008)) are designed to evaluate the long-term chemical behavior in the environment. However, these tests are expensive, technically sophisticated, and time-consuming (e.g., use of several different tests and the use of ${ }^{14} \mathrm{C}$-labeled chemicals) (Nyholm et al. 1992; Ericson 2010), limiting the tests' use to a very small number of substances. There is, therefore, a gap between ease of use and biodegradability assessment, hence the necessity for the community of environmental stakeholders to strike a balance to assess biodegradation between Ready Biodegradation Tests and simulation.

This problem has been recurrent for 60 years; assessing chemical persistence in the environment is not straightforward and better screening tests are needed (Boethling et al. 2009). In all test classes, the substance-specific properties are known, which provide indications of potential environmental behavior (e.g., solubility, volatility). What is unknown is the number of microorganisms that are potentially present in the environment and competent to degrade the released molecules under in situ conditions. If the growth of a group of bacterial species with the tested substance as the only carbon source is the very principle of biodegradability assessment, the environmental fate of the molecule would be linked to the likelihood to be in contact with these specific bacterial communities. With this in mind, we suggest performing biodegradability assessment for soluble substances following a stochastic approach. This new concept, which is denoted by ProbaBio, for Probability of Biodegradation, connects the likelihood to inoculate specific microbial degraders from different environments in a given biodegradation test with the probability for a chemical to be biodegraded. Thus, according to the ProbaBio approach (Thouand et al. 2011), persistence is not regarded as a simple intrinsic substance property, but as the capability of various environmental samples to degrade a substance under realistic exposure scenarios and conditions (e.g., regional and local wastewater treatment plants (WWTPs), soil and water types, climate changes). Using a screening platform and a probabilistic approach, ProbaBio provides probability profiles of microbial degradation that are obtained under more realistic environmental conditions relative to cell density and temperature.

\section{Materials and methods}

An overview of the strategy used to provide the ProbaBio proof of concept is presented in Fig. 1.

\section{Sampling collection and inoculum preparation}

The environmental samples were selected to be representative of the many natural compartments in which chemicals can be discharged (see supplementary data Fig. 1). Activated sludge, freshwater, soils, and seawater were sampled using sterile equipment and were stored at $4{ }^{\circ} \mathrm{C}$ during transportation to the laboratory. An aliquot of each sample was stored at $4{ }^{\circ} \mathrm{C}$, and all samples were immediately prepared. Activated sludge was first filtered through $500-\mu \mathrm{m}$ nylon cloth, sonicated three times $(0.400 \mathrm{~kJ})$, and filtered through $5-\mu \mathrm{m}$ nylon cloth. Freshwater and seawater were centrifuged $(6400 \mathrm{~g}, 10 \mathrm{~min}$, $4{ }^{\circ} \mathrm{C}$ ) to concentrate microorganisms. Pellets were resuspended in sterile mineral medium and were filtered through $5-\mu \mathrm{m}$ nylon cloth. Soils were incubated in sterile phosphatebuffered saline (PBS) solution $(v / v)$ under agitation for $2 \mathrm{~h}$ at $4{ }^{\circ} \mathrm{C}$. The mixture was then filtered through $500-\mu \mathrm{m}$ nylon cloth, sonicated three times $(0.400 \mathrm{~kJ})$, and filtered through $5-\mu \mathrm{m}$ nylon cloth. Then, all inocula were washed, centrifuged,

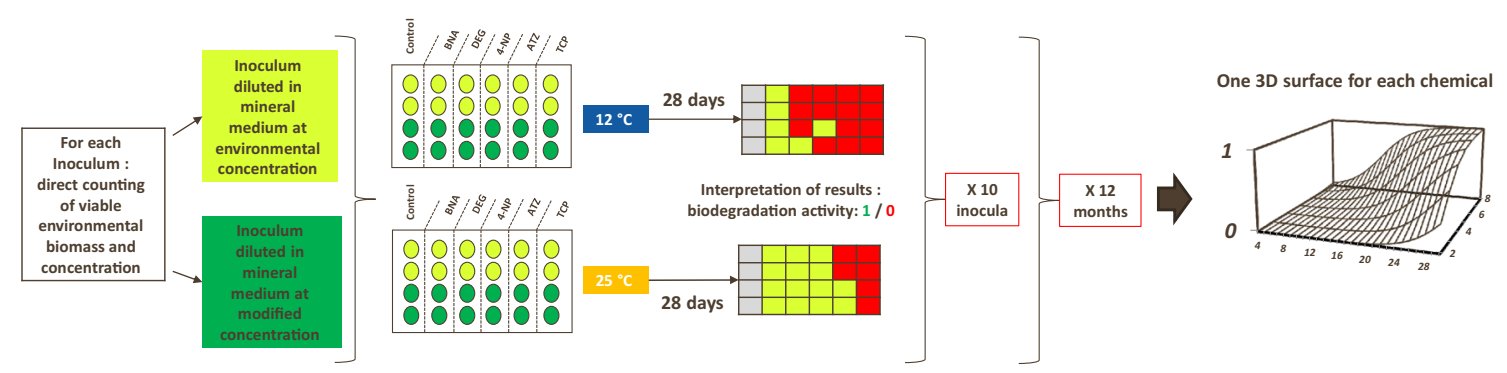

Fig. 1 Global overview of the ProbaBio strategy. Ten environments are sampled. Bacteria are extracted, pretreated, and inoculated at two different concentrations separately in duplicate in 24-well microplates with non-invasive sensors for dissolved oxygen monitoring. Microplates are incubated at two different temperatures during 28 days 
and resuspended in sterile mineral solution $\left(\mathrm{MgSO}_{4}, 10^{-2} \mathrm{M}\right)$ three times.

\section{Total cell count}

The concentrations of living cells in the environmental and concentrated samples were measured by LIVE/DEAD BacLight Bacterial Viability Kit for microscopy under an epifluorescence microscope with a $\times 400$ objective according to the manufacturer's instructions (Invitrogen, France).

\section{Chemical preparation}

Solutions of sodium benzoate (abbreviated BNA; CAS 53232-1, Fluka 71,300), 4-nitrophenol (abbreviated 4-NP; CAS 100-02-7, Sigma 73,560), diethylene glycol (abbreviated DEG; CAS 111-46-6, Sigma 93,171), 2,4,5-trichlorophenol (abbreviated TCP; CAS 95-95-4, Sigma 36,513), atrazine (abbreviated ATZ; CAS 1912-24-9, Sigma 45,330), and glyphosate (abbreviated GLY; CAS 1071-83-6, Sigma 45, 521 ) were prepared in the culture medium for biodegradation assessment described in the OECD 301B guidelines at a final concentration of $10 \mathrm{mg}$ of carbon per liter.

\section{High-throughput and miniaturized system for biodegradability assessment}

The platform developed in our laboratory for biodegradation screening tests was used (Cregut et al. 2013). The platform is based on non-invasive sensors for dissolved oxygen monitoring in 24-well plates (PreSens, Ge) filled with $2.5 \mathrm{ml}$ of chemical stock solutions. Inoculum samples were diluted and added at both the environmental concentration and 10 times the environmental concentration for a maximum concentration of
$10^{8}$ cells $\mathrm{ml}^{-1}$. Environmental samples with a cell concentration higher than $10^{8}$ cells $\mathrm{ml}^{-1}$ were diluted. All samples were inoculated in duplicate. A control inoculum used to assess the endogenic consumption of $\mathrm{O}_{2}$ (mineral culture medium + microorganisms) was included for each condition and environment. Microplates were sealed and incubated at 12 and $25^{\circ} \mathrm{C}$. The evolution of dissolved oxygen was measured online for 28 days. A positive biodegradation result was validated when (i) $\mathrm{O}_{2}$ consumption in the inoculum control was inferior to $0.5 \mathrm{mg} \mathrm{l}^{-1}$ and (ii) $\mathrm{O}_{2}$ consumption of the assay was greater than 10 times the maximal value observed in the negative control at any time point. An example is given in Fig. 2. Note that a positive biodegradation result does not necessarily mean that an ultimate biotransformation of tested substances has occurred. With this high-throughput system, distinction between primary and ultimate biodegradation is not possible. Consequently, positive results and models discussed in this work are based on the observation of an event of biodegradation and are not necessarily linked to a complete mineralization of chemicals introduced in the assays.

\section{Statistical methods}

The proportions of positive results, which were observed under different experimental conditions, were compared using either the chi-squared or the likelihood ratio test, according to the requirements for performing each of these statistical methods. The Mann-Whitney-Wilcoxon test was used to test the effect of the number of cells inoculated on the outcome of each biodegradation test. Logistic regression analysis was performed on the binary outcomes of the biodegradation tests to examine the effects of the explanatory variables on the biodegradability. To this end, the probability of observing a positive result for a biodegradation test was expressed as a logistic

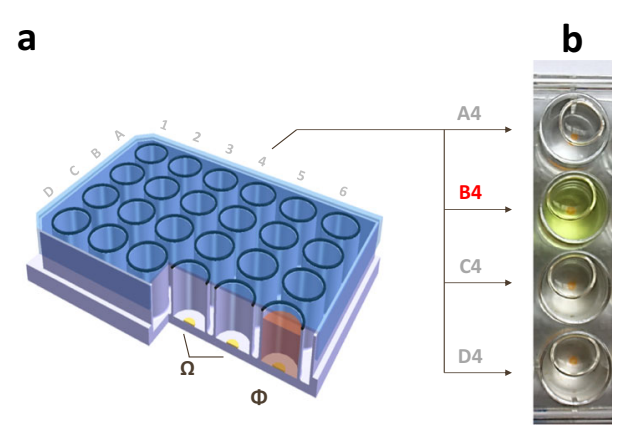

Fig. 2 High-throughput and miniaturized system for biodegradability assessment. a Twenty-four-well microplates with non-invasive sensors for dissolved oxygen monitoring $(\Omega)$ were filled with $2.5 \mathrm{ml}$ of chemical stock solution plus inocula $(\Phi)$. b $4-\mathrm{NP}$ can be considered as biodegradable in only three of four cases (4-NP is yellow at $10 \mathrm{mg}$ of carbon per liter and $\mathrm{pH}=7.4$ and is clear when biodegraded). c Concentration of dissolved oxygen for 28 days for 4-NP. The negative control (bacterial inoculum + mineral culture medium) consumption of

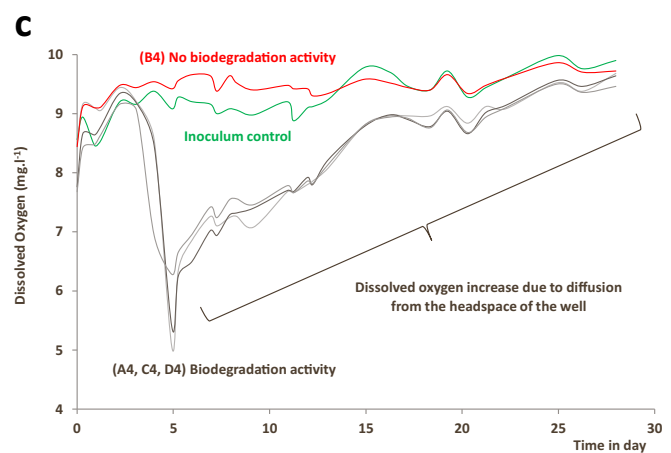

oxygen is very low (less than $0.5 \mathrm{mg} \mathrm{l}^{-1}$ ). The consumption of oxygen in the assays (carbon source (4-NP) + bacterial inoculum + mineral culture medium) from A4, C4, and D4 is more than 10 times the negative control and can be attributed to the biodegradation of 4-NP (validated by the color loss in these same wells). The consumption of oxygen in the assay B4 is equal to the inoculum control and cannot be considered as positive (validated by the yellow color in these same wells). $\mathrm{pH}$ has been checked at the end of each experiment and is equal to 7.4 
function of both the temperature and the cell density of each sample, including an interaction term between these two variables. Three-dimensional surface plots of the fitted logistic regression models were used to profile the potential environmental persistence of a chemical in terms of the probability of biodegradation expressed as a function of the temperature and the cell density. The volume under each surface was used as a risk indicator of potential persistence in environmental conditions. This volume is expressed as a number called the ProbaBio Index (PBI), which can be used as an index characterizing the biodegradability of a given molecule. Moreover, calculating the variance of the estimator of the probability of biodegradation allowed us to construct lower and upper confidence 3D surface plots and subsequently compute confidence limits at a predetermined probability level of the PBI which is associated with the chemical considered. All of the computations were performed using the statistical package $\mathrm{R}$ (version 2.12.2; Bell Laboratories, Lucent Technologies; http://www.r-project.org).

\section{Results and discussion}

Thousands of biodegradation tests were performed with five compounds, in different environments throughout a year, at different biomass concentrations and incubation temperatures, providing a matrix of 4244 tests.

\section{Sampling network and data set presentation}

From November 2012 to October 2013, samples from 10 environments ( 3 WWTPs, 3 soils, 3 freshwater, and 1 seawater; see supplementary data Fig. 1) were collected each month. Temperatures and the numbers of cells were subject to very large variations with differences observed intraand inter-environments (Fig. 3). For all of these environments, throughout the year, the average temperature measured was

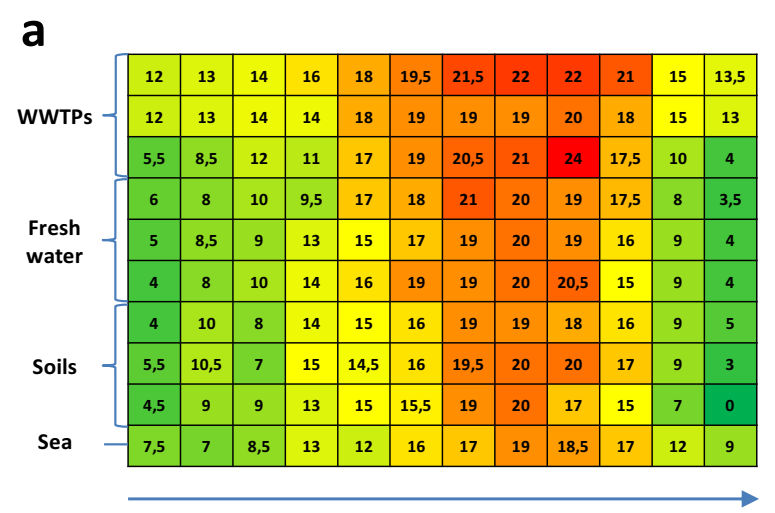

12 months from January to December
$13.7{ }^{\circ} \mathrm{C}$ with a maximum at $24{ }^{\circ} \mathrm{C}$ (month of June in a WWTP) and a minimum of $0{ }^{\circ} \mathrm{C}$ (month of December in a soil). The number of cells also evolved throughout the year but was mainly different from one environment to another, with a difference in the annual average of $10^{3}$ cells ml $^{-1}$ between the most concentrated activated sludge (WWTP Nantes

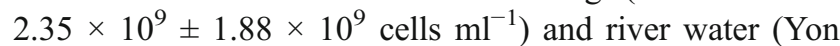
$1.04 \times 10^{6} \pm 7.18 \times 10^{5}$ cells ml $^{-1}$ ).

Biodegradation of BNA, 4-NP, DEG, TCP, and ATZ was assessed in these environments in different conditions using a 1 (chemical tested in duplicate) $\times 10$ (environments separately) $\times 12$ (months) $\times 4$ (variations of in vitro parameters) factorial experimental design (see "Materials and methods" section and Fig. 1). For each molecule, more than 840 biodegradation tests were analyzed (of a total of 960 tests, approximately $12 \%$ were uninterpretable due to carbon contamination in the inoculum control). Among these five molecules (Table 1), two are described as readily biodegradable (BNA and DEG) by the European Chemical Agency (ECHA). Our results are consistent with ECHA for BNA (100 \% of the tests are positive). For DEG, biodegradation activity was observed in only $60.88 \%$ of the tests performed. 4-NP is described as inherently biodegradable and well known to fail RB tests, which is consistent with an observed variable biodegradation activity depending on the condition tested $(79.02 \%$ of the tests were positive). Regarding ATZ and TCP, which are characterized as persistent by ECHA, they were biodegradable in 4.72 and $0 \%$ of the cases for ATZ and TCP, respectively. As shown by these results, the biodegradation test outcomes for certain molecules may not always be positive (DEG, 4-NP, and ATZ) when tested in several environments under microbial realistic exposure scenarios and conditions.

\section{Influence of exposure scenarios and conditions}

For the three molecules showing variable biodegradation results, 4-nitrophenol, diethylene glycol, and atrazine, the

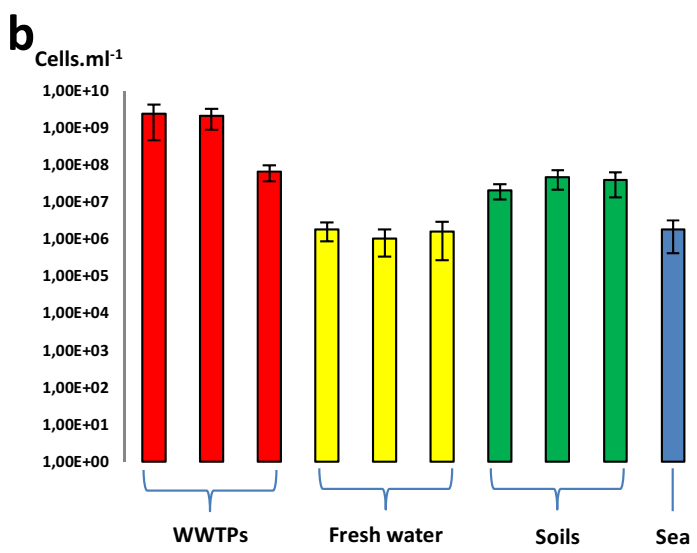

Fig. 3 Environmental variability of the sampled sites from November 2012 to October 2013 . a In situ temperature $\left({ }^{\circ} \mathrm{C}\right)$ from coldest $\left(4{ }^{\circ} \mathrm{C}\right.$, green) to hottest $\left(24^{\circ} \mathrm{C}, \mathrm{red}\right)$ and $\mathbf{b}$ average bacterial concentration (cells $\left.\mathrm{ml}^{-1}\right)$ in environments sampled 
Table 1 Summary of the chemicals used in the proof of concept of ProbaBio with $n=\sim 850$ tests or with $n=80$ tests

Chemicals (CAS no.)

\begin{tabular}{ll}
\hline Sodium benzoate (532-32-1) & 10,000 to 100,000 \\
Diethylene glycol (111-46-6) & 100,000 to $1,000,000$ \\
4-Nitrophenol (100-02-7) & Intermediate use only \\
Atrazine (1912-24-9) & Intermediate use only \\
2,4,5 trichlorophenol (95-95-4) & Intermediate use only \\
Diethylene glycol (111-46-6) & 100,000 to $1,000,000$ \\
Glyphosate (1071-83-6) & No data available
\end{tabular}

sampling location of the bacterial inoculum has a significant impact on the success or failure of the test (Fig. 4a). Positive test percentages are highly dependent on the inoculum sampling location for 4-NP $(P<0.001)$ with some environments being more efficient in degrading this compound (freshwater Yon $95.83 \%$, WWTP Roche sur Yon $92.68 \%$, urban soil $91.66 \%)$. These observations are the same for DEG $(P<0.001)$ and ATZ $(P<0.001)$, although the most effective environments are different (DEG WWTP Roche sur Yon and freshwaters; ATZ WWTP Roche sur Yon and river Yon). We also show (Fig. 4b) for 4-NP and ATZ that the season in which the inoculum was sampled has a significant impact on the success or failure of a test. This impact is only slightly significant for 4-NP $(P=0.035)$, with a maximum of positive tests observed in winter and spring, whereas ATZ was mostly biodegraded in May and August $(P<0.001)$, with very low percentages in other months.

\section{Influence of temperature and cell density}

The probability of the biodegradation of DEG, 4-NP, and ATZ is related not only to the intrinsic chemical properties but also to the environmental variables such as the geographical conditions (e.g., in situ cell density and temperature). In an attempt to study the effect of environmental variation on the outcome of the biodegradation tests for these molecules, the influence of cell concentration and incubation temperature was evaluated in the laboratory (Fig. 4c). A statistically significant increase of positive results was observed between the tests performed at 12 and $25^{\circ} \mathrm{C}$ for each of the three molecules $(P<0.001)$. Concretely, 9.66, 1.39, and 2.85 times more positive results were observed at $25^{\circ} \mathrm{C}$ than at $12{ }^{\circ} \mathrm{C}$ for, respectively, ATZ, 4-NP, and DEG. The number of cells inoculated at the beginning of the tests also had a statistically significant effect on biodegradation. The higher the cell concentration is, the higher the probability of biodegradation is (ATZ $P=0.003$, 4-NP $P<0.001$, DEG $P<0.001$ ). Moreover, the number of positive results observed with enhanced concentration of the inoculums is estimated to be 1.7, 1.17, and 1.28 times the corresponding number at the environmental concentration, for ATZ, 4-NP, and DEG, respectively.
It is then clear that the strategies (RBT, simulation) of the evaluation of the biodegradation process, which is a significant part of the environmental persistence assessment, cannot be based on the outcome of a laboratory test that does not take into account changes in terms of bacterial diversity, quantity, and climatic conditions. Indeed, these parameters naturally evolve over time and space and directly impact the communities of microorganisms that are potentially degrading chemical compounds.

\section{Modeling biodegradability}

The probability of biodegradation has been expressed as a logistic function of the temperature and the cell density, including an interaction term between these two variables. Thus, the reduced fitted logistic model for the probability of biodegradation, which was computed from more than 840 tests with binary outcomes, is shown in Fig. $4 d$ for each molecule examined. These three-dimensional surfaces plots model the probability to obtain a positive biodegradation test as a function of the inoculated quantity of cells from 10 different sites (effect of quantity and diversity) and the temperature (climatic conditions). From left to the right, the surfaces shown in Fig. $4 \mathrm{~d}$ evolve from top to bottom according to the global probability of obtaining a positive test for each of the chemicals. According to the probabilities obtained from the fitted model, BNA (used as positive control in several biodegradation test guidelines) tests are positive under any experimental conditions, i.e., within the range of values considered for the temperature and the cell density. The probabilities of biodegradation corresponding to DEG, 4-NP, and ATZ are shown to depend on the values of the explanatory variables. In other words, for these three chemicals, the outcome of a biodegradability test is dependent on both the bacterial inoculum and the temperature, with the highest probabilities at high temperature and high cell quantity. Moreover, the two explanatory variables do not have the same impact in terms of the probability of biodegradation according to the chemical tested. For DEG, a $1 \log$ variation of the cell concentration has the same effect on biodegradability as a variation of $3.5^{\circ} \mathrm{C}$. Similarly, for 4-NP and ATZ, a $1 \log$ variation of cell 


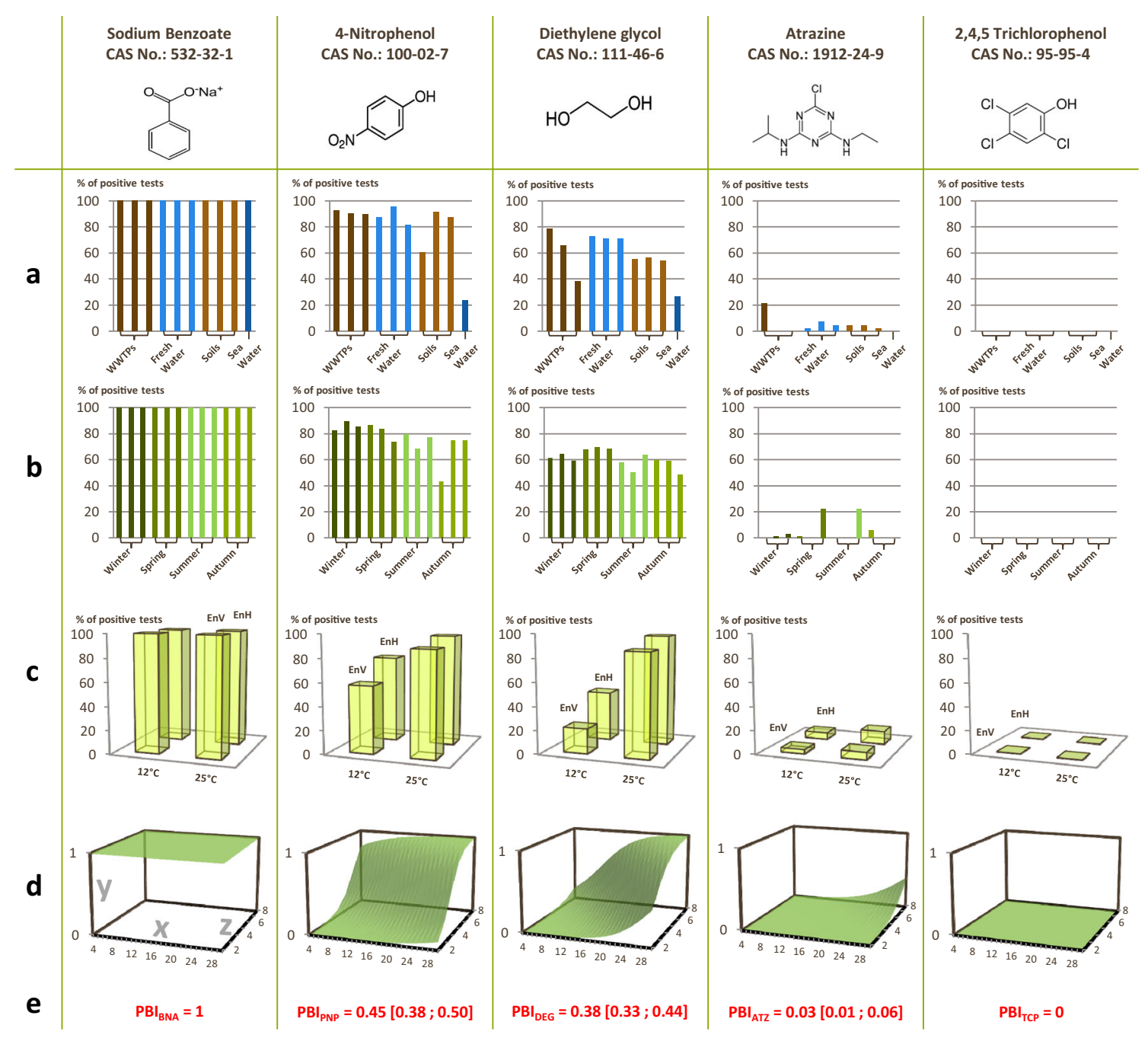

Fig. 4 ProbaBio proof of concept. a, b Influence of the origin of the inocula (wastewater treatment plans (WWTPs)) and period of sampling on the percentage of positive biodegradation tests over all other varying parameters. $\mathbf{c}$ Influence of the incubation temperature $\left(12\right.$ or $\left.25^{\circ} \mathrm{C}\right)$ and cell density (environmental (EnV) test inoculated depending on the in situ environmental concentration, enhanced $(\mathrm{EnH})$ test inoculated at 10 times environmental concentration for a maximum of $10^{8}$ cells $\mathrm{ml}^{-1}$ ) on the percentage of positive biodegradation tests. $\mathbf{d}$ Three-dimensional surfaces

plots of the fitted logistic regression models to describe the probability of biodegradation $(y)$ as a function of the temperature ( $x$ between 4 and $30{ }^{\circ} \mathrm{C}$ ) and the cell density ( $z$ between $10^{2}$ and $10^{8}$ cells $\mathrm{ml}^{-1}$ ). e The estimated ProbaBio Index (PBI) varying from 1 to 0 , according to decreasing values of the probability of microbial degradation, with $95 \%$ confidence limits. PBI values are calculated as the volume located under the three-dimensional surface plot of the logistic regression models

concentration is equivalent to 7.5 and $4{ }^{\circ} \mathrm{C}$, respectively. For TCP, which had no positive tests, the estimated probability of biodegradation is uniformly equal to zero.

\section{Surfaces and ProbaBio index to profile biodegradation under environmental conditions}

According to the fitted logistic regression models, the probability of biodegradation calculated from various environmental samples and values of the temperature can be extended to define a profile of microbial degradation in the environment. For example, the potential risk for BNA to be persistent when released in the environment is expected to be very low because the corresponding model predicts that all of the results of the biodegradation tests are positive. This, however, is not the case for DEG and 4-NP because the positive test predictions are low outside of the most favorable conditions (no biodegradation activity predicted in 28 days). Hence, the microbial degradation profiles of these two molecules point toward potential environmental persistence. This is especially true for ATZ. With very few conditions that result in positive tests, the environmental fate of this compound may exhibit persistence in certain conditions. A high risk of persistence in certain environmental compartments is expected. With a downward flat surface, the profile of the microbial degradation of TCP is the lowest, which means that this compound could be highly persistent. 
Based on the decrease of the probability to obtain a positive biodegradation test and consequently the lowering of surfaces, microbial degradation profiles can be ranked. A risk indicator of potential persistence in environmental conditions has been determined by calculating the volume under each surface. This volume is expressed as a number called the PBI and can be used as an index characterizing the biodegradability of a given molecule. This number, which can be calculated with $95 \%$ confidence limits, ranges from 1 to 0 , according to whether the molecule considered is potentially highly biodegradable (low environmental persistence expected) to potentially poorly biodegradable (high environmental persistence expected), respectively (Fig. 4e). Thus, the estimated PBI is equal to 1 for BNA, $0.44(0.38,0.50)$ for 4 NP, $0.38(0.32,0.44)$ for DEG, $0.03(0.01,0.06)$ for ATZ, and 0 for TCP. This allows a ranking of the chemicals, in terms of their biodegradability, from readily biodegradable to not biodegradable in the environment, BNA $>4$ $\mathrm{NP}>\mathrm{DEG}>\mathrm{ATZ}>\mathrm{TCP}$.

\section{From the proof of concept to a reduced experimental designed tool to profile biodegradability in the environment}

The high number of biodegradation tests, which were performed to establish the ProbaBio concept (more than 800 per chemical), was used to perform a global study of the effects and interactions of the environmental variables and to ensure the reliability of the fitted regression models. Nevertheless, this number was too high for current laboratory applications. Therefore, to make it accessible to industry, scientific community, and environmental agencies, a reduction of the experimental design was examined. To this end, we decided to profile the biodegradation of DEG, as an example, by

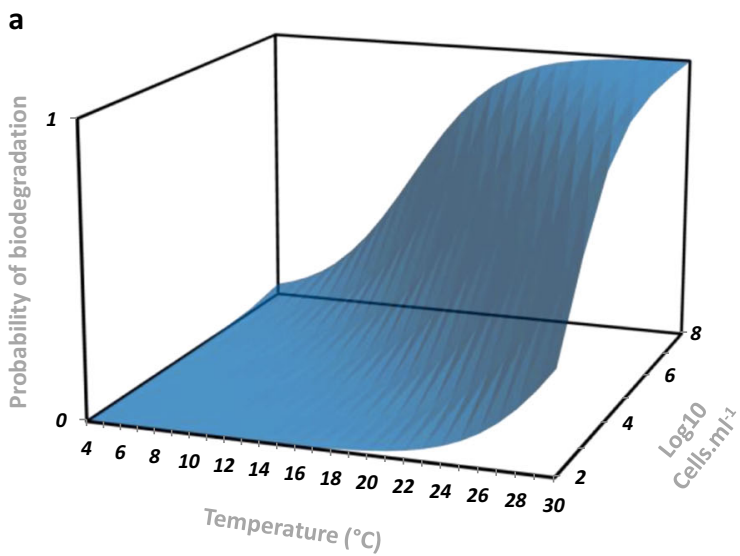

Fig. 5 Optimized ProbaBio concept applied to diethylene glycol. a Three-dimensional surface plot of the fitted logistic regression model $(n=80)$ to profile the potential environmental persistence of DEG in terms of the probability of biodegradation expressed as a function of the means of only 40 tests, which were performed in duplicate $(n=80)$. Thus, during the month of September 2014, the same 10 environments were sampled to inoculate four microplates of the high-throughput and miniaturized system for biodegradability assessment at 2 cell concentrations and 2 incubation temperatures. Here, it is worth pointing out that similar analyses could also have been carried out by randomly selecting $n=80$ out of the full set of test results. Indeed, "in silico" analyses may offer a convenient way to demonstrate how reducing the number of tests would affect the results and their associated uncertainties. The results are shown in Fig. 5a. With $n=80$, the percentage of positive tests was $58.75 \%$. This result is in close agreement and is not statistically significantly different from the first study with $n=844$ (positive tests $=60.88 \%$ (chi-squared test, $P=0.93)$ ). Moreover, the coefficient of determination calculated between the corresponding results, which were obtained from the logistic regression models with $n=80$ and $n=844$, is equal to 0.97 . Consequently, the surface used to profile the biodegradation of DEG with $n=80$ is similar to the first surface, thus showing the same influence of the variable temperature and cell concentration on the probability of biodegradation (Fig. 5b). Here, a 1 log variation of the cell concentration is equivalent to $3.2{ }^{\circ} \mathrm{C}$ in terms of ProbaBio $\left(3.5{ }^{\circ} \mathrm{C}\right.$ with $n=844)$. The ProbaBio Index with $95 \%$ confidence is $\mathrm{PBI}=0.32(0.18,0.48)$ and $\mathrm{PBI}=0.38(0.32,0.44)$ for $n=40$ and $n=854$, respectively. Both the environmental biodegradation profiles and the PBI of the DEG, established from 80 or more than 800 tests, describe the same risk of potential persistence under certain environmental conditions. Seen another way, it may be possible to achieve a reliable ProbaBio approach with only 40 tests, performed in duplicate. This means that a lab-scale application of ProbaBio could be performed in a lightweight form.

b

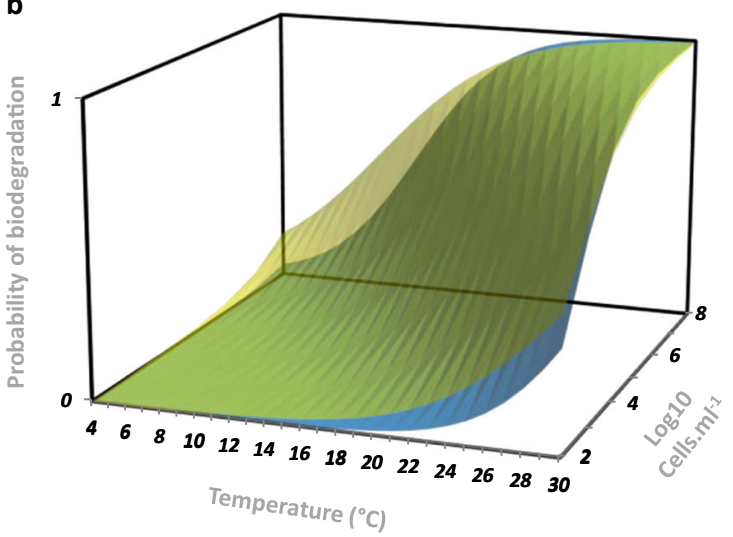

temperature (between 4 and $30^{\circ} \mathrm{C}$ ) and the cell density (between $10^{2}$ and $10^{8}$ cells $\mathrm{ml}^{-1}$ ). b Comparison of the three-dimensional surface plots characterizing the environmental profiles of DEG with $n=80$ (blue surface) and $n=844$ (yellow surface) 


\section{ProbaBio: case study with glyphosate}

Glyphosate $[N$-(phosphomethyl) glycine] is the active ingredient of the world's biggest selling herbicide. Studies and reviews on its environmental fate have been numerous in the last 30 years, and bibliographic findings often describe the same difficulties to assess glyphosate biodegradation due to geographic and climatic influences, among other influences, on the environmental fate of this molecule (Carlisle and Trevors 1988; Giesy et al. 2000; Annett et al. 2014). In view of these conclusions, we performed a lab-scale application of ProbaBio on glyphosate with the same reduced experimental design as DEG above. Of a total of 80 tests, $11.25 \%$ were positive (mostly, $44 \%$, with an inoculum from a farm wastewater treatment plant; other inocula were from a freshwater sample and soils). According to the estimated probability of positive biodegradation tests calculated by means of the fitted logistic regression model (Fig. 6), the maximum probability was 0.48 at $30^{\circ} \mathrm{C}$ and $10^{8}$ cells ml ${ }^{-1}$. Apart from these favorable conditions (i.e., high temperatures and high concentrations of microorganisms), the glyphosate biodegradation probabilities are low. Indeed, the biodegradation profile, as given by the three-dimensional surface plot, highlights a potential hazardous biodegradation profile in the environment, which is highly dependent on in situ conditions. The probability of the Biodegradation Index with $95 \%$ confidence limits is estimated at $0.036(0.005,0.15)$, which suggest that glyphosate has the same hazardous profile as atrazine. Our conclusions are in full agreement with those of other scientific reviews and articles on the same subject; glyphosate can be considered biodegradable (Imfeld et al. 2013), but its biodegradation profile can quickly change depending on the geographical (Borggaard and Gimsing 2008) and climatic (Helander et al. 2012) variations and can present the risk of persistence.

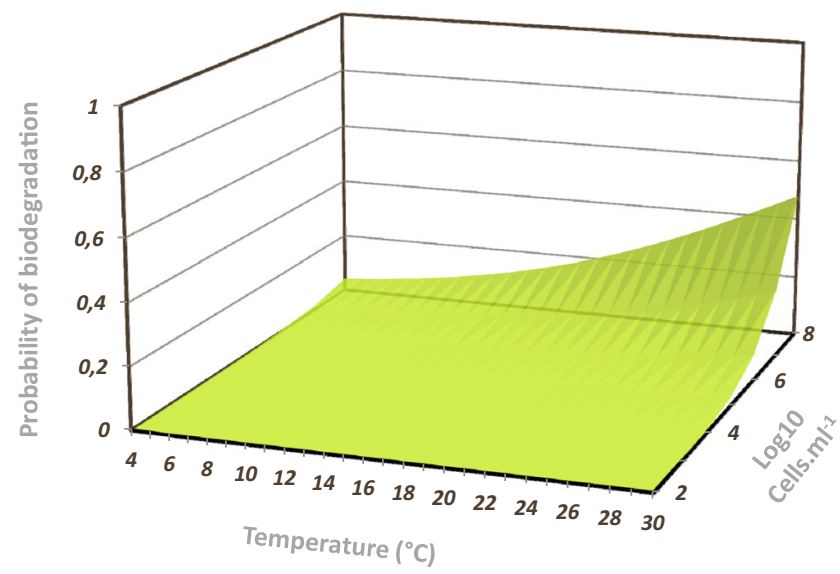

Fig. 6 Microbial biodegradation profile of glyphosate according to the optimized ProbaBio concept $(n=80)$

\section{Advantages, contributions, and limits of the ProbaBio concept}

For biodegradation tests realized according to OECD standard procedures from the guidelines 301 , assays are incubated at only one temperature level (commonly $22 \pm 2{ }^{\circ} \mathrm{C}$ ) and are inoculated with only one quantity of cells (between $10^{4}$ and $10^{8}$ cells $\mathrm{ml}^{-1}$ ) sampled generally from a single environment. When tested under these conditions, the probability for DEG to be biodegraded varies from $0.22\left(10^{4}\right.$ cells ml $\left.^{-1}\right)$ to 0.96 $\left(10^{8}\right.$ cells $\left.\mathrm{ml}^{-1}\right)$, whereas the probability for glyphosate to be biodegraded increases from 0 to 0.26 . However, with tests allowing higher initial cell concentrations, both DEG and glyphosate could be considered biodegradable in some environments. Therefore, what would not have been measured with standard tests clearly appeared with ProbaBio; the environmental profiles of these two chemicals are not constant. Moreover, the estimated surfaces are not always at the top, as was the case for BNA. Indeed, the environmental profiles change in time and space, and the risks of persistence may appear, according to the very low probabilities of the positive biodegradation tests, which can be observed when either the temperature and/or the cell density decrease. However, such low levels of temperature and cell density are realistic environmental conditions for widespread chemicals. It must be emphasized that what is possible to observe from compiling several scientific studies may be highlighted in less than 1 month of testing with ProbaBio. Moreover, this technically simple concept is not static and can be significantly improved. In this regard, it must be pointed out that extrapolating beyond the tested values is model dependent and may thus be questionable in terms of the accuracy of the predictions made. Indeed, the choice of a logistic function to model biodegradation, with values ranging from 0 to 1 as the bounds corresponding to low and high values, respectively, of both the temperature and the cell density, is liable to restrict the shape of the fitted model considerably. In particular, potential abrupt changes in microbial community structure and activity (e.g., depending to low and very low temperatures) cannot be accounted for with the present approach. Still, one should keep in mind that our approach is just a first attempt to model biodegradation, in the sense that it is essentially an exploratory research work, which is intended to be improved, namely, for use in the context of a more systematic (screening) application. Indeed and as said before, the number of biodegradation tests which was used in the present study is too high for current laboratory and/or industrial purposes. Thus, reducing the number of these tests (e.g., $n=40$ ) may advantageously allow to optimize the standard experimental design for further applications. This can be attained for instance by increasing the number of levels and, subsequently, the range of the values which are tested (e.g., increasing the levels of incubation temperature to achieve greater accuracy). Other variables can also 
be integrated into the model according to the chemical behavior and mode or conditions of use (e.g., focus on specific geographical area). The biological degradation processes in environmental conditions are multifactorial and are subsequently very complex phenomena because other influencing variables are additionally present in natural compartments. Furthermore, the concentrations of some chemical molecules may be low, and they are often mixed with organic material or other chemicals or metabolized by co-metabolism. These additional variables are not yet included in ProbaBio. Finally, ProbaBio still retains many of the technical limitations common to ready biodegradability standardized procedures (e.g., testing of poorly soluble chemicals, biotransformation of microbial communities during inoculum pre-treatment processes; Goodhead et al. 2014). Consequently, ProbaBio has not been designed to replace OECD guidelines but provide a complementary approach for chemical biodegradability assessment using different sources of microbial inocula and temperature regimes. Probes integrated in a high-throughput system to quantify and qualify ultimate biodegradation have also to be designed and tested in ProbaBio conditions. With a system allowing an estimation of the half-life, a combination of our concept with existing quantitative structure-activity relationship (QSAR)/quantitative structure-biodegradation relationship (QSBR) would improve, through the generation of data, the prediction of environmental fate. Testing this concept with more chemicals could help better define the limits and conceivable solutions to increase the reliability of this tool.

\section{Conclusions}

Predicting the behavior and the environmental fate of newly synthesized chemicals remains a priority for the community of environmental stakeholders. Thus, the management of the ever-increasing flow of chemicals requires appropriate concepts to assess the chemicals' risk during the screening stages. Regarding the behavior and the evaluation of intrinsic persistence, there are many tools available for laboratories to establish the potential for biodegradation. However, predicting the probability of biodegradation by assuming given environmental conditions is not a simple task. Ready Biodegradation Tests are not adapted to addressing the quantity and complexity of environmental variables. To our knowledge, this is the first time a strategy in biodegradation assessment bridges the gap between experimental ease of use and probabilistic approach of biological persistence. The ProbaBio approach presented in this study is a new concept that allows stakeholders to perform biodegradation tests under environmentally more realistic conditions. As a concept, ProbaBio combines the exposure scenario and probabilities of biodegradation using a miniaturized high-throughput system and logistic regression analysis. As a result, ProbaBio profiles microbial degradation, in the sense that it both indicates the potential risk of environmental persistence and allows the quantification of this risk, as shown for the tested molecule glyphosate after only 1 month of testing. Furthermore, including this strategy in the decision tree of environmental persistence assessment for new molecules could help industrial, scientific, and environmental agencies to better understand and hence manage the risk of environmental persistence.

\section{References}

Ahtiainen J, Aalto M, Pessala P (2003) Biodegradation of chemicals in a standardized test and in environmental conditions. Chemosphere 51: 529-537. doi:10.1016/S0045-6535(02)00861-5

Annett R, Habibi HR, Hontela A (2014) Impact of glyphosate and glyphosate-based herbicides on the freshwater environment. J Appl Toxicol 34:458-479. doi:10.1002/jat.2997

Aronson D, Boethling R, Howard P, Stiteler W (2006) Estimating biodegradation half-lives for use in chemical screening. Chemosphere 63:1953-1960. doi:10.1016/j.chemosphere.2005.09.044

Bartholomew GW, Pfaender FK (1983) Influence of spatial and temporal variations on organic pollutant biodegradation rates in an estuarine environment. Appl Environ Microbiol 45:103-109

Boethling R, Fenner K, Howard P, et al. (2009) Environmental persistence of organic pollutants: guidance for development and review of POP risk profiles. Integr Environ Assess Manag 5:539-556. doi:10.1897/IEAM_2008-090.1

Borggaard OK, Gimsing AL (2008) Fate of glyphosate in soil and the possibility of leaching to ground and surface waters: a review. Pest Manag Sci 64:441-456. doi:10.1002/ps. 1512

Carlisle SM, Trevors JT (1988) Glyphosate in the environment. Water Air Soil Pollut 39:409-420. doi:10.1007/BF00279485

Cregut M, Jouanneau S, Brillet F, et al. (2013) High throughput and miniaturised systems for biodegradability assessments. Environ Sci Pollut Res Int. doi:10.1007/s11356-013-2236-2

ECHA (2011) Guidance on information requirements and chemical safety assessment part B: hazard assessment.

ECHA (2014) Guidance on information requirements and chemical safety assessment part $\mathrm{C}: \mathrm{PBT} / \mathrm{vPvB}$ assessment.

ECHA R.7B (2014) Guidance on information requirements and chemical safety assessment chapter R.7b: endpoint specific guidance.

Ericson JF (2010) Evaluation of the OECD 314B activated sludge dieaway test for assessing the biodegradation of pharmaceuticals. Environ Sci Technol 44:375-381. doi:10.1021/es902205r

Federle TW, Gasior SD, Nuck BA (1997) Extrapolating mineralization rates from the ready $\mathrm{CO}_{2}$ screening test to activated sludge, river water, and soil. Environ Toxicol Chem 16:127-134. doi:10.1002 letc.5620160205

Fenner K, Scheringer M, Hungerbühler K (2004) Prediction of overall persistence and long-range transport potential with multimedia fate models: robustness and sensitivity of results. Environ Pollut 128: 189-204. doi:10.1016/j.envpol.2003.08.028

Forney LJ, Liu W-T, Guckert JB, et al. (2001) Structure of microbial communities in activated sludge: potential implications for assessing the biodegradability of chemicals. Ecotoxicol Environ Saf 49:40 53. doi:10.1006/eesa.2001.2034

Giesy JP, Dobson S, Solomon KR (2000) Ecotoxicological risk assessment for roundup ${ }^{\circledR}$ herbicide. In: Ware DGW (ed) Reviews of environmental contamination and toxicology. Springer, New York, pp. $35-120$ 
Goodhead AK, Head IM, Snape JR, Davenport RJ (2014) Standard inocula preparations reduce the bacterial diversity and reliability of regulatory biodegradation tests. Environ Sci Pollut Res Int 21: 9511-9521. doi:10.1007/s11356-013-2064-4

Helander M, Saloniemi I, Saikkonen K (2012) Glyphosate in northern ecosystems. Trends Plant Sci 17:569-574. doi:10.1016/j. tplants.2012.05.008

Howard PH, Stiteler WM, Meylan WM, et al. (1992) Predictive model for aerobic biodegradability developed from a file of evaluated biodegradation data. Environ Toxicol Chem 11:593-603. doi:10.1002 letc.5620110502

Imfeld G, Lefrancq M, Maillard E, Payraudeau S (2013) Transport and attenuation of dissolved glyphosate and AMPA in a stormwater wetland. Chemosphere 90:1333-1339. doi:10.1016/j. chemosphere.2012.04.054

Mackay D, Webster E (2005) Environmental persistence of chemicals. Environ Sci Pollut Res 13:43-49. doi:10.1065/espr2006.01.008

Martiny JBH, Bohannan BJM, Brown JH, et al. (2006) Microbial biogeography: putting microorganisms on the map. Nat Rev Microbiol 4: 102-112. doi:10.1038/nrmicro1341

Nyholm N (1991) The European system of standardized legal tests for assessing the biodegradability of chemicals. Environ Toxicol Chem 10:1237-1246. doi:10.1002/etc.5620101002

Nyholm N, Damborg A, Lindgaard-Jørgensen P (1992) A comparative study of test methods for assessment of the biodegradability of chemicals in seawater-screening tests and simulation tests. Ecotoxicol Environ Saf 23:173-190. doi:10.1016/0147-6513(92 )90057-A

OECD (1992) Test no. 301: ready biodegradability. Organisation for Economic Co-operation and Development, Paris

OECD (2008) Test no. 314: simulation tests to assess the biodegradability of chemicals discharged in wastewater. Organisation for Economic Co-operation and Development, Paris

Pizzo F, Lombardo A, Manganaro A, Benfenati E (2013) In silico models for predicting ready biodegradability under REACH: a comparative study. Sci Total Environ 463-464:161-168. doi:10.1016/j. scitotenv.2013.05.060
Ranjard L, Dequiedt S, Chemidlin Prévost-Bouré N, et al. (2013) Turnover of soil bacterial diversity driven by wide-scale environmental heterogeneity. Nat Commun 4:1434. doi:10.1038 /ncomms 2431

Raymond JW, Rogers TN, Shonnard DR, Kline AA (2001) A review of structure-based biodegradation estimation methods. J Hazard Mater 84:189-215. doi:10.1016/S0304-3894(01)00207-2

Thouand G, Durand M-J, Maul A, Blok H (2011) New concepts in the evaluation of biodegradation/persistence of chemical substances using a microbial inoculum. Front Microbiotechnol Ecotoxicol Bioremediation 2:164. doi:10.3389/fmicb.2011.00164

Thouand G, Friant P, Bois F, et al. (1995) Bacterial inoculum density and probability of para-nitrophenol biodegradability test response. Ecotoxicol Environ Saf 30:274-282. doi:10.1006/eesa.1995.1031

UE (2006) Regulation (EC) No 1907/2006 of the European Parliament and of the Council of 18 December 2006 concerning the Registration, Evaluation, Authorisation and Restriction of Chemicals (REACH), establishing a European Chemicals Agency, amending Directive 1999/45/EC and repealing Council Regulation (EEC) No 793/93 and Commission Regulation (EC) No 1488/94 as well as Council Directive 76/769/EEC and Commission Directives 91/155/EEC, 93/67/EEC, 93/105/EC and 2000/21/EC. Official Journal of the European Union L136, 1-280

Van Ginkel CG, Haan A, Luijten ML, Stroo CA (1995) Influence of the size and source of the inoculum on biodegradation curves in closedbottle tests. Ecotoxicol Environ Saf 31:218-223

Vazquez-Rodriguez G, Goma G, Rols JL (2012) Toward a standardization of the microbial inoculum for ready biodegradability testing of chemicals. International Water Association, pp 43-46

Vink JPM, Van Der Zee SEATM (1997) Pesticide biotransformation in surface waters : multivariate analyses of environmental factors at field sites. Water Res 31:2858-2868

Zogg GP, Zak DR, Ringelberg DB, et al. (1997) Compositional and functional shifts in microbial communities due to soil warming. Soil Sci Soc Am J 61:475. doi:10.2136 /sssaj1997.03615995006100020015x 